\title{
Prevalence and Genotypic Identification of Human Papillomavirus Infec- tion in a Population from Northwestern Spain
}

\author{
C. Rodríguez-Cerdeira ${ }^{*}, 1$, R. Chillón ${ }^{1}$, S. Díez-Moreno ${ }^{1}$ and A. Guerra-Tapia ${ }^{2}$ \\ ${ }^{\text {I} D e r m a t o l o g y ~ D e p a r t m e n t . ~ C H U V I ~ \& ~ U n i v e r s i t y ~ o f ~ V i g o . ~ V i g o, ~ S p a i n ~}$ \\ ${ }^{2}$ Dermatology Department. Hospital Doce de Octubre. Madrid, Spain
}

\begin{abstract}
We present a cross-sectional observational descriptive study that was carried out on a reference population of 84,000 women from Northwestern Spain in order to study the prevalence of human papillomavirus (HPV) and its repercussion in genital cancer. The group of patients for this study was of 436 patients with ages ranging from 16 to 80 years old. Three samples were taken from each woman in this group from the cervix and vagina, which was, later, processed using PCR techniques. The most predominant serotype was PHPV-16, followed by HPV-31. Type 1 CIN (low grade cervical intraepithelial neoplasia) (LG-CIN) was the most predominant.
\end{abstract}

\section{INTRODUCTION}

Epidemiological relationships were established more than 100 years ago between precursory lesions of cervical cancer and risky sexual behaviour. For various decades medicine has, unsuccessfully, tried to find the relationships between the agents responsible for sexually transmitted infections with this neoplasia. At the beginning of the 80's the association between HPV and cervical cancer was discovered. In recent years, multiple clinical, epidemiological and laboratory investigations have demonstrated that the main etiopathogenic agent in precursory lesions for cervical cancer is HPV [1]. The papillomaviruses are viruses which are grouped together due to their tumourigenicity and the homogeneity of their DNA, together with the fact that they affect vertebrates. At present there are over 100 known genotypes of HPV; with each type showing its own particular tropism for specific anatomical sites, with infections of the skin and oral and respiratory tract mucosa being common. Over 30 genotypes can infect the anogenital tract. The International Agency for Cancer Research (IACR), which belongs to the WHO, classifies the 16 and 18 viral types of HPV as carcinogenic and, the 31 and 33 types as probably carcinogenic. In later data, taken from 11 studies carried out in various countries, in which the relationship between the cervix and HPV was studied, 15 viral strains were identified as being oncologenically high-risk (HR-HPV) $(16,18,31,33,35,39$, $45,51,52,56,58,59,68,73$ and 82); types 26,53 and 66 were classified as probable HR-HPV; and types 6, 11, 40, 42, 43, 44, 54, 61, 70, 72, 81 and CP6108 were classified as low risk strains (LR-HPV). Persistent infection by HPV is considered to be the main causative agent for cervical cancer and other anogenital cancers [2].

*Address correspondence to this author at the Department of Dermatology, CHUVI, Meixoeiro Hospital \& University of Vigo, 36200 Vigo, Galicia, Spain; Tel: 0034600536114; Fax: 0034986276416;

E-mail: aristoteles_cerdeira@yahoo.es
Cervical cancer is, at present, the second most frequent cancer in women in the world and the first in women in developing countries, with an estimated global incidence of 470,000 new cases per year. Moreover, it causes approximately 273,000 deaths per annum [3]. Over $80 \%$ of cases are from developing countries. In these countries fewer than $50 \%$ of the women with cervical cancer survive for more than 5 years, while survival at 5 years is approximately $66 \%$ in developed countries [4].

\section{MATERIALS AND METHODS}

The study was carried out on 436 women with ages ranging from 16 to 80 years old. All patients were given cytology with triple samples from the cervix and vagina. Samples which proved positive for HPV were classified, via PCR, into distinct existing HPV serotypes, through the COBAS system AmpliPrep ${ }^{\circledR}$. A total of 13 patients were rejected as the samples were incorrectly taken or because they did not have clear infection by papillomavirus. The aims of the study were: to know the distribution of the genotypes of high-risk HPV in endocervical samples as well as the prevalence of multiple infections. To establish the association of high-risk HPV genotypes as a unique infectious genotype or in multiple infections. To determine the possible incidence of the HPV vaccine in the prevention of these infections in our environment.

Statistic analysis: This was carried out using SPSS-14. A descriptive analysis of all the study variables collected was performed using mean and standard deviation for the quantitative variables and frequencies and percentages for the qualitative variables, studying the prevalence and incidence rates.

For association between qualitative variables the Chisquared test was used.

Finally we shall do a survival analysis using Cox regression analysis, considering cervical cancer as a response variable and those which are related to the event as predictive or protective variables. 


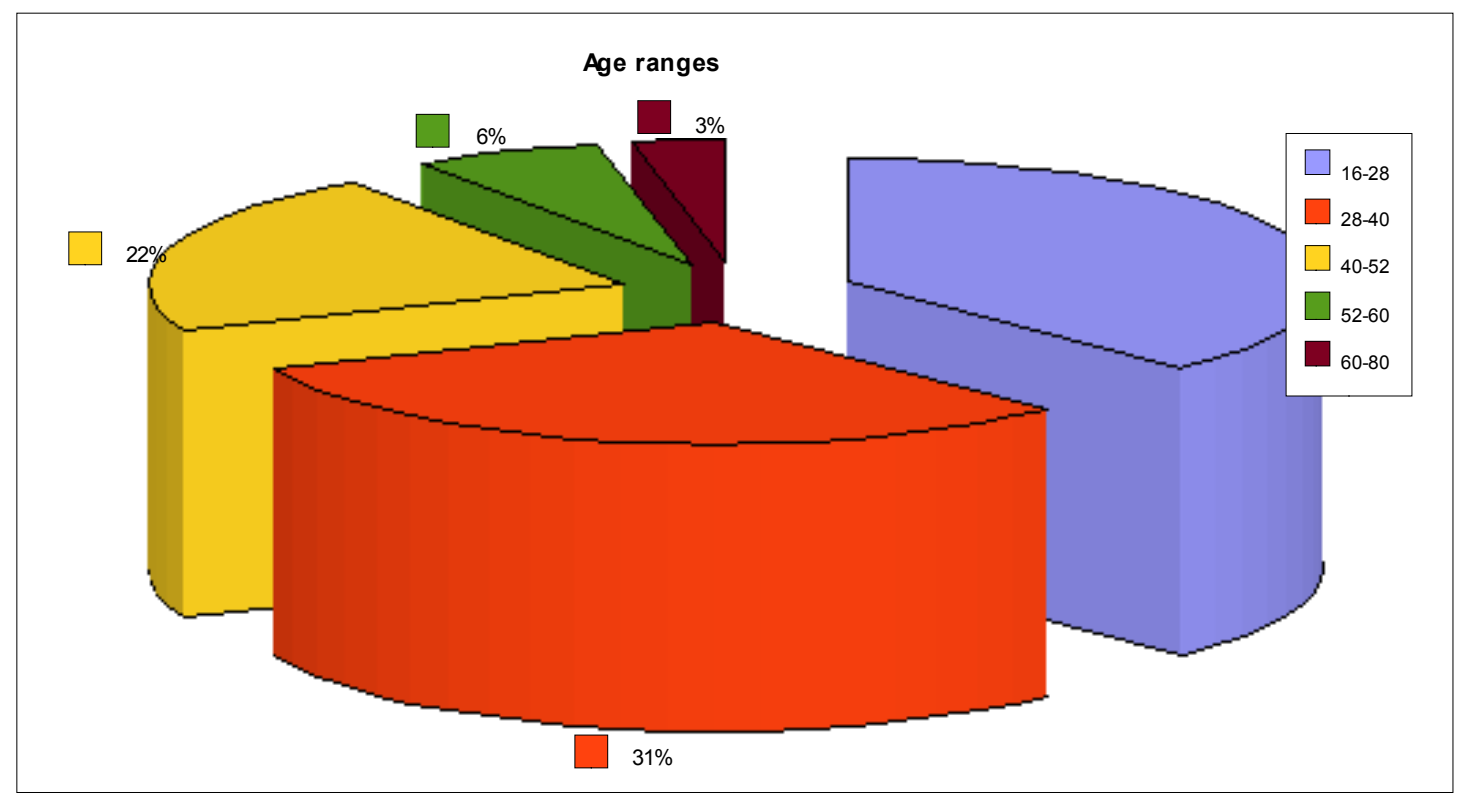

Fig. (1). Age distribution (436 patients).

\section{RESULTS}

We studied a total of 436 women. The mean age was $34.87 \pm 1.8$ years old. The age range distribution is shown graphically in Fig. (1), where we observe that, as the age increases, contagion by HPV decreases. All patients were given a triple vaginal sampling and a later cytological analysis. From the 436 patients examined, 125 were found to be positive for HPV, and 13 were rejected since the sample was incorrectly taken or since they did not have an evident HPV infection (Fig. 2). All samples that were seen to be positive through cytological screening were classified into distinct HPV serotypes by PCR. In the population studied, we observed a $29 \%$ incidence of HPV infection, among which the highest percentage (37.6\%) corresponded to women of 16 to 28 years old.

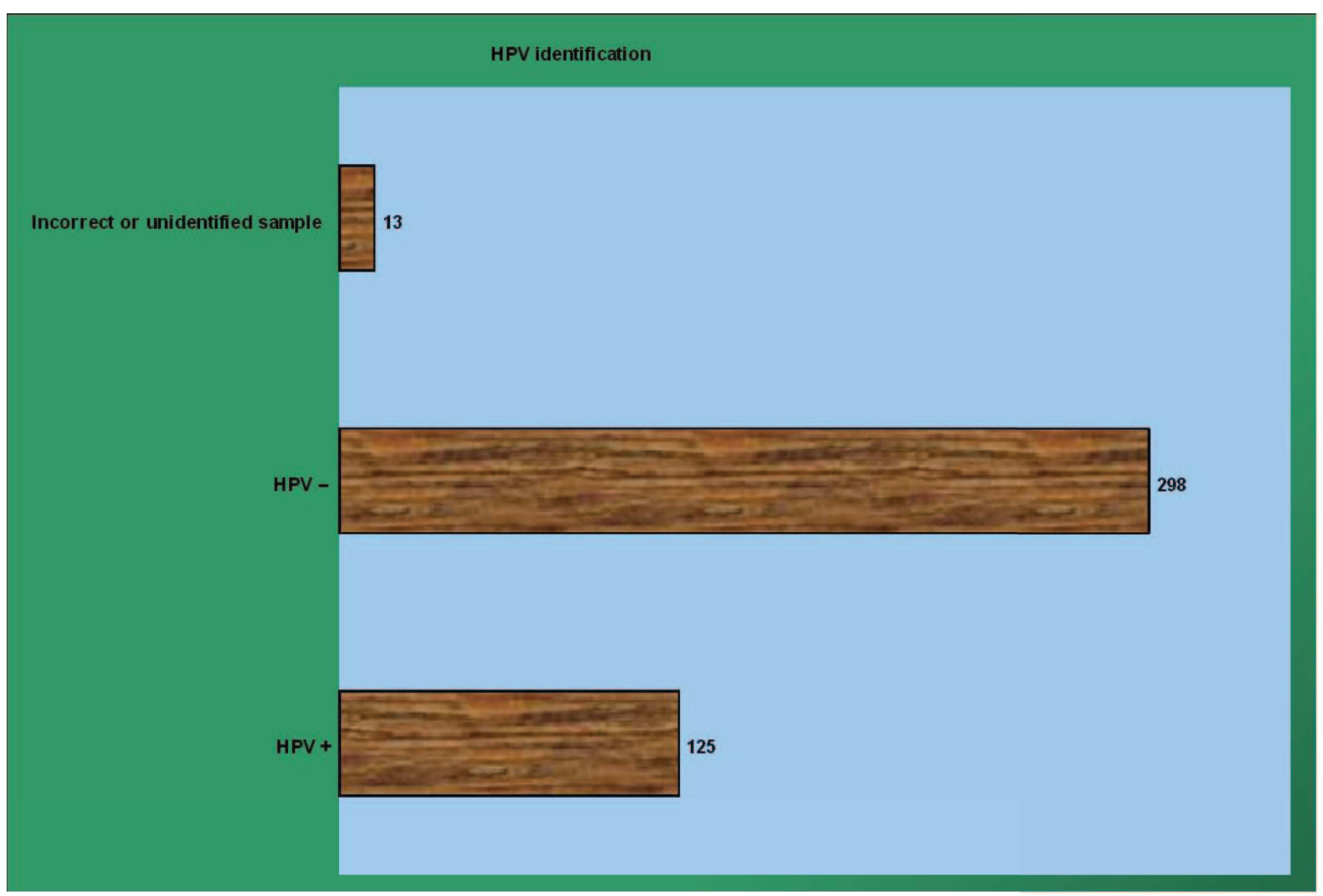

Fig. (2). Detection of the HPV infection in a number of 436 patients. 


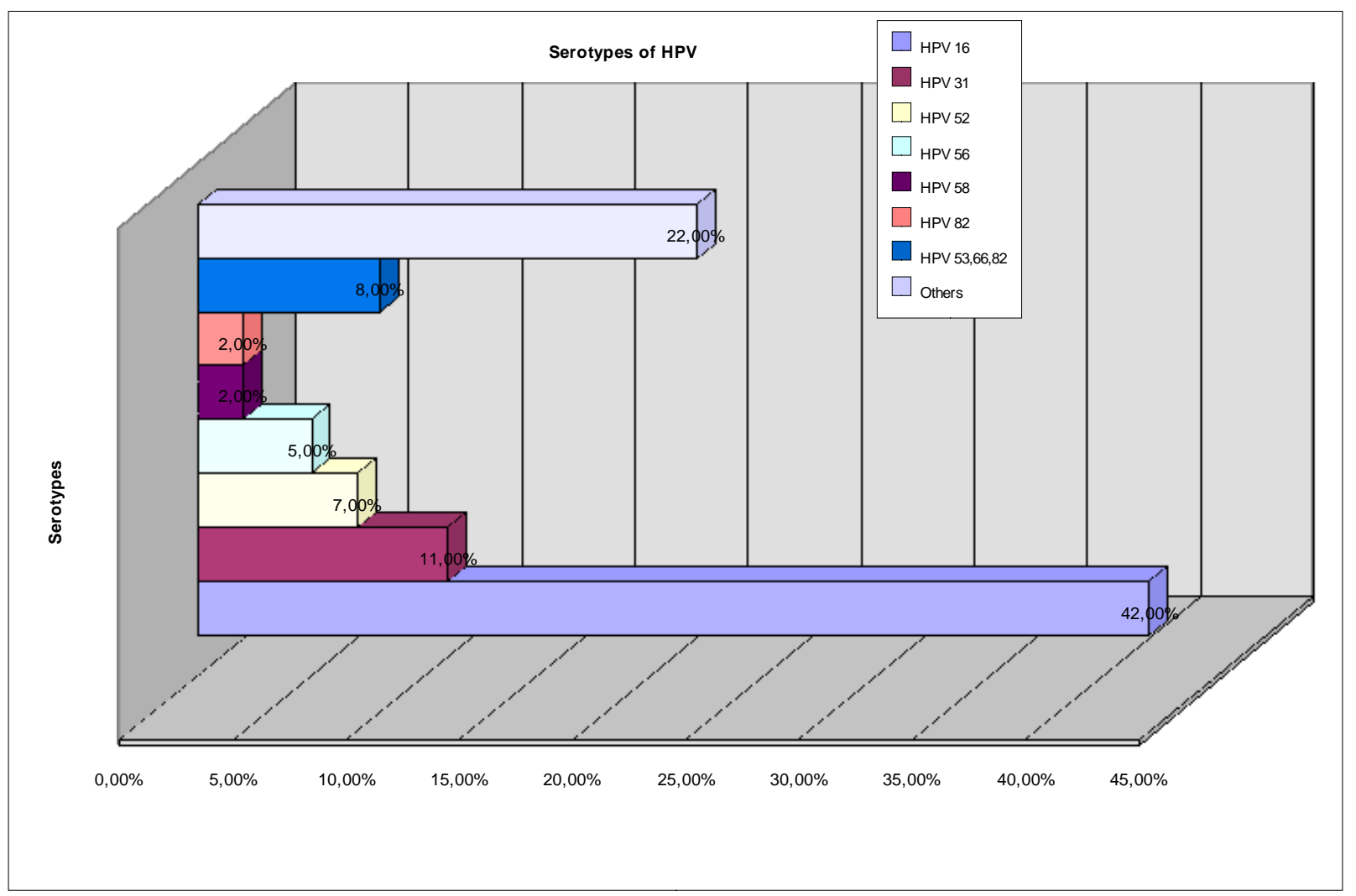

Fig. (3). Percentage of distinct serotypes of HPV (125 patients).

Type 16 showed the greatest prevalence. The percentage of other serotypes found in the sample are given in Fig. (3).

Another important finding was the histopathologic correlation carried out on the abnormal cervical cytologies. Type CIN I (low grade-cervical intraepithelial neoplasia) (LG-CIN) was the most predominant. In second place, we found CIN II (high grade-cervical intraepithelial neoplasia) (HG-CIN) type lesions in two patients of 22 and 67 years of age.

Three patients, of 27, 49 and 55 years of age, developed type CIN III (HG-CIN). The various serotypes of HPV that existed are given in Fig. (3).

We would, furthermore, like to highlight the frequency of co-infection with other germs. Associated candidiasis was observed in $9.7 \%$ of the cases. Finally, we highlight mixed infection by candida and gardenerella vaginalis, which are generally found together in younger patients, and which are observed in the sample with a $9.7 \%$ frequency for each of them. series using high PCR, 90.7\% of cervical-uterine carcinomas have DNA from HPV, and, 100\% of the cases have been confirmed with exhaustive histological examinations, and in the majority of the intraepithelial lesions of the lower genital tract [6].

Type 16 showed the greatest prevalence, on being detected in half of the cases. A similar situation was found in our series. The sequence that, possibly, exists in the high grade lesions is associated with the infection by the high-risk strains of HPV, although this was not exclusively the case. Co-infections with HPV-16 and HPV-58 seem to be particularly dangerous [7, 8]. The majority of these infections are transitory, being produced in $70-90 \%$ of infected women, which generally coincides with the period of greater sexual activity [9].

It has been observed that women under 35 years of age are more susceptible to acquiring genital infections with oncogenic virus, which disappear in the majority of cases; as opposed, to women over 35 years old where the lesion often per- 
sion to invasive carcinoma being $2.5 \%$. Among the $15-30 \%$ of women found positive for DNA from HR-HPV with normal cytology on admission there was development to types CIN II (HG-CIN) or CIN III (HG-CIN) in the following four years. In another broad literature review on the natural history of type CIN I (LG-CIN), regression occurred in $60 \%$ of patients, persistence in $30 \%$; progression to type CIN III (HG-CIN) in $10 \%$, and invasion in $1 \%$ [12]. In prospective studies, the risk of developing CIN II/III (HG-CIN) during follow-up of CIN I (LG-CIN) confirmed by biopsy is $9-16 \%$ [13].

Our patients show CIN I ( LG-CIN) in $66 \%$ of cases, CIN II (HG-CIN) in 2\%; and CIN III (HG-CIN) in $3 \%$. We have not yet observed progression to invasive carcinoma in any of our patients during follow-up.

Factors associated with viral class, genetics and related to sexual behaviour of the woman or environmental have been described. Viral determinants for progression include: the viral type, the viral load per cellular unit, phylogenetic variants and integration with cellular DNA. Possible genetic factors include markers for genetic susceptibility, factors which regulate the cellular humoral immunological responses to infection by HPV, HLA, and p53, among many others. Among the environmental factors for acquisition of HPV in women are: the age at which they experienced the first coitus, multiple partners, or partner with high-risk behaviour, high parity, poor hygiene and smoking. Other cofactors described are the existence of other sexually transmitted diseases, such as hepatitis B, C, Chlamydia trachomatis, herpes virus, syphilis, HIV, since a group with higher risk of progression of the invasive carcinoma is the HIV positive group, especially those who do not respond to or do not carry out antiretroviral treatment, encouraging an immunosuppressive state [14]. In our study we only found co-infections with candida albicans and gardenerella vaginales. None of our patients was HIV positive.

As HPV vaccination is now included in the vaccination schedule, after this preliminary study we propose to carry out a follow-up of all these women, vaccinating as many women as possible, where deemed economically viable, and studying again, afterwards, the behaviour of the distinct serotypes of HPV, above all, those that are no longer the most prevalent $[15,16]$.

\section{CONCLUSIONS}

The prevalence of HPV in the female lower genital tract still shows high levels, with a maximum in prevalence in young women, confirming a decrease in infection in middle age. Nonetheless, the virus shows an outburst in postmenopausal women. The HPV-16 serotype was the most highly prevalent (27\%), followed by HPV-31 (11\%), HPV-52 (7\%), HPV-56 (5\%), HPV-58 (2\%). Multiple infections are present in $38 \%$ of the patients and reduce with age. The HPV16 and 31 serotypes show similar behaviour in both multiple infections or when acting as sole infective serotypes.
With the use of vaccines against HPV, especially the tetravalent vaccine, in the short or medium-term there will be a lower rate of abnormal cytological results in vaccinated women, which is based on the expected preventive potential, which may oscillate between 50 to $70 \%$ of the cases. Furthermore, with the use of the vaccine, in combination with present screening programs, we expect to reach a $92 \%$ reduction in the annual number of cases of cervical cancer and other associated pathologies in our country.

\section{REFERENCES}

[1] Bosch FX, Manos MM, Munoz N, et al. Prevalence of human papillomavirus in cervical cancer: a worldwide perspective. J Natl Cancer Inst 1995; 87: 796-802.

[2] Rodríguez-Cerdeira MC, Alba A, Bravo G, Alcántara R. Human papilloma virus infection and its repercussion in female genital pathology. Piel 2007; 22: 1171-80.

[3] Parkin DM, Bray F, Ferlay J, Pisani P. Estimating the world cancer burden: Globocan 2000. Int J Cancer 2001; 94: 153-6.

[4] Yang BH, Bray FI, Parkin DM, Sellors JW, Zhang ZF. Cervical cancer as a priority for prevention in different world regions: an evaluation using years of life lost. Int J Cancer 2004; 109: 418-24.

[5] Castellsagué X, Díaz M, de Sanjose S, et al. Worlwide human papillomavirus etiology of cervical adenocarcinoma and its cofactors implications for screening and prevention. IARC Multicenter Cervical Cancer Study Group. J Natl Cancer Inst 2006; 98: 303-15.

[6] Krambeck WM, Cadidé RM, Dalmarco EM, de Cordova CM. HPV detection and genotyping as an earlier approach in cervical cancer screening of the female genital tract. Clin Exp Obstet Gynecol 2008; 35: $175-8$.

[7] Trottier H, Mashmud S, Costa MC, Sobrinho JP, Duarte-Franco E, Rohan TE. Human papillomavirus infections with multiple types and risk of cervical neoplasia. Cancer Epidemiol Biomarkers Prev 2006; 15: 1274-80.

[8] Rodríguez-Cerdeira MC, Gonzalez-Guerra E, Guerra-Tapia A. Vulvar cancer. Más Dermatol 2008; 4: 5-13.

[9] Herrero R, Hildesheim A, Bratti C, et al. Population-based study of human papillomavirus infection and cervical neoplasia in rural Costa Rica. J Natl Cancer Inst 2000; 92: 464-74.

[10] Kjaer SK, van den Brule AJ, Paull G, et al. Type specific persistence of high human papillomavirus (HPV) as indicator of high grade cervical squamous intraepithelial lesions in young women, population based prospective follow up study. BMJ 2002; 325: 572-8.

[11] Rodríguez-Cerdeira C, de Argila D, A Guerra. Tumores malignos. In: Guerra Tapia A, Ed. Manual y atlas de las enfermedades de la vulva. Barcelona: Glosa 2006; 261-83.

[12] Ostor AG. Natural history of cervical intraepithelial neoplasia: a critical review. Int J Gynecol Pathol 1993; 12: 186-92.

[13] Weaver MG, Abdul-Karim FW, Dale G, Sorensen K, Huang YT. Outcome in mild and moderate cervical dysplasias related to the presence of specific human papillomavirus types. Mod Pathol 1990; 3: $679-83$.

[14] Rodríguez Cerdeira C, Carpintero Feijoo Y, Alcántara Cáceres R Vilata Corell JJ. Sífilis adquirida. En: Vilata Corell JJ, Ed. Venereología: aspectos epidemiológicos y clínicos de las infecciones transmitidas por vía sexual. Madrid: Aula Médica 2005; 51-61.

[15] Rodríguez-Cerdeira MC, Alba A, Vilata JJ. Development of new human papillomavirus vaccines. Piel 2007; 22: 51-3.

[16] de Sanjosé S, Alemany L, Castellsagué X, Bosch FX. Human papillomavirus vaccines and vaccine implementation. Womens Health (Lond Engl) 2008; 4: 595-604. 